\title{
Efficacy and Safety of Sunitinib in Japanese Patients with Metastatic Renal Cell Carcinoma
}

\author{
_- Sunitinib for Japanese Patients Financial Disclosures: None
}

\author{
Takahito Suyama $^{1}$, Takeshi Ueda ${ }^{2}$, Satoshi Fukasawa ${ }^{2}$, Atsushi Komaru², Masayuki Kobayashi ${ }^{2}$, \\ Yukio Naya ${ }^{1}$, Naoki Nihei ${ }^{1}$, Tomohiko Ichikawa ${ }^{1}$
}

${ }^{1}$ Department of Urology, Chiba University Graduate School of Medicine, Chiba, Japan; ${ }^{2}$ Prostate Center and Division of Urology, Chiba Cancer Center, Chiba, Japan.

Email: urolccc@yahoo.co.jp

Received April 10 ${ }^{\text {th }}$ 2011; revised June 16 ${ }^{\text {th }}, 2011$; accepted June $23^{\text {rd }}, 2011$.

\begin{abstract}
Objective: This study aim to assess the efficacy and safety of sunitinib in Japanese patients with metastatic renal cell carcinoma (mRCC) in general clinical practice. Patients and Methods: Non-selected fifty eight Japanese patients with $m R C C$ were treated with sunitinib. Overall survival $(O S)$ and time to treatment failure $(T T F)$ were estimated. Response rate and safety profiles were also assessed. Results: Partial response, stable disease, and progressive disease were observed in 13 (22.4\%), 26 (44.8\%), and 19 (32.8\%) patients, respectively. The median TTF was 5.4 months, and the median OS was 11.2 months. In the prior nephrectomy group, the median TTF was 9.0 months, and the median OS was 16.4 months. In the non-nephrectomy group, the median TTF was 1.1 months, and the median OS was 2.8 months. The most frequently occurring Grade 3/4 adverse events (AEs) were anorexia (17.2\%), fatigue (12.1\%), thrombocytopenia (13.8\%), and anemia (12.1\%). Conclusions: Sunitinib has a favorable risk/benefit profile in Japanese mRCC patients with a history of nephrectomy.
\end{abstract}

Keywords: Renal Cell Carcinoma, Metastasis, Sunitinib, Adverse Event

\section{Introduction}

Renal cell carcinoma (RCC) is the most common cancer of the kidney [1]. RCC presents with metastatic disease in up to $30 \%$ of patients, and recurrence develops in $\sim 40 \%$ of patients treated for a localized tumor [2]. Although RCC currently constitutes $<5 \%$ of adult malignancies, the incidence of RCC is increasing, especially in developed countries [1]. Metastatic RCC (mRCC) is a highly chemoresistant disease, and until 2005, immunotherapy was recommended as a first-line treatment. However, immunotherapy was effective only in selected patients, up to $20 \%$, and median overall survival (OS) was $\sim 1$ year [3]. The advent of molecular-targeted drugs has resulted in a reduction in the use of immunotherapy as first-line treatment in mRCC.

RCCs are highly vascularized tumors that overexpress a number of growth factors and their receptors, including vascular endothelial growth factor (VEGF), platelet-derived growth factor (PDGF), and transforming growth factor, secondary to the inactivation of the von
Hippel-Lindau tumor suppressor gene [4,5]. These ligands and receptors are involved in the autocrine stimulation of tumor cell growth, as well as the paracrine stimulation of neovascular or stromal fibroblast growth that supports tumor expansion [2,5]. Thus, inhibitors or antagonists of these pathways (e.g., bevacizumab [6], sorafenib [7], and sunitinib [8]) have demonstrated marked anti-tumor activity in patients with $\mathrm{mRCC}$, particularly those with the clear-cell form of the disease.

Sunitinib malate (SUTENT; Pfizer Inc., New York, NY, USA) is an oral, multitargeted tyrosine kinase inhibitor that acts on VEGF receptors 1 - 3 [9], PDGF receptors [9, 10], and KIT [10]. In a pivotal Phase III trial in patients with $\mathrm{mRCC}$, sunitinib demonstrated a significantly longer progression-free survival (PFS) time (11 vs. 5 months, $\mathrm{P}<0.001)$ and higher objective response rate $(47 \%$ vs. $12 \%)$ than interferon (IFN)- $\alpha$ alone as first-line treatment [8]. Sunitinib also showed a strong tendency toward prolonged OS (26.4 vs. 21.8 months, $\mathrm{P}=0.051)$ when compared with IFN- $\alpha$ alone, despite the crossover to sunitinib in $33 \%$ of patients with IFN- $\alpha$ after disease 
progression [11].

However, patients included in this trial had to satisfy strict eligibility criteria that induced good performance status, could have no serious co-morbidities, and had to have adequate organ function. In addition, only patients with clear-cell histology were eligible for the study [8]. Although sunitinib has been universally prescribed to patients with $\mathrm{mRCC}$, few studies have examined the efficacy and safety of this agent in daily clinical practice, especially in patients of Asian ethnicity [12-15]. Here, we assessed the efficacy and safety of sunitinib in Japanese patients with $\mathrm{mRCC}$ in general clinical practice.

\section{Patients and Methods}

\subsection{Patient Characteristics}

We conducted a retrospective analysis of 58 consecutive and non-selected patients with mRCC treated with sunitinib between July 2008 and October 2010 at the Division of Urology, Chiba Cancer Center and at the Department of Urology, Chiba University Hospital, Chiba, Japan. Patient characteristics are shown in Table 1. Medical records were retrospectively reviewed for clinicopathologic characteristics such as age, sex, histology, Eastern Cooperative Oncology Group performance status, prior treatments, sites of metastasis, laboratory findings, tumor progression, and patient survival. Patients were included in this study regardless of histology and prior treatment, as well as history of nephrectomy. All patients were confirmed as having RCC either pathologically by nephrectomy (44 patients) or by biopsy (14 patients). Pretreatment evaluation consisted of a complete history and physical examination, laboratory tests, computed tomography (CT) scan and/or magnetic resonance imaging, and total body bone scan. Of the 58 patients included, 8 patients received sunitinib after failed cytokine therapy (i.e., with IFN- $\alpha$ and interleukin-2); 25 patients received first-line sunitinib treatment, and 24 patients received sunitinib after failed sorafenib (23 patients), everolimus (1 patient), or temsirolimus (1 patient) treatment with/ without cytokine therapy. Risk groups were based on the 5 risk factors included in the Memorial Sloan-Kettering Cancer Center (MSKCC) risk criteria [16].

\subsection{Treatment}

Sunitinib was administrated orally at a starting dose of 50 $\mathrm{mg}$ once daily in the morning for 4 weeks, followed by a 2 -week resting period ( $4 / 2$ schedule). Sunitinib dose reduction was allowed depending on the type and severity of adverse events (AEs). Patients underwent a physical examination on day 1 and day 28 of every treatment cycle. Complete blood cell counts and serum chemistry tests were performed on days 1, 14, and 28 of each cycle.
Table 1. Patient characteristics .

\begin{tabular}{cc}
\hline Characteristics & $\mathrm{n}(\%)$, total $=58$ \\
\hline Male & $48(82.8)$ \\
Median age, years (range) & $65(32-79)$ \\
ECOG performance status & \\
0 & $36(62.1)$ \\
1 & $17(29.3)$ \\
2 & $4(6.9)$ \\
3 & $1(1.7)$ \\
Histological type & $42(72.4)$ \\
Clear & $16(27.6)$ \\
Non-clear & \\
Prior nephrectomy & $44(75.9)$ \\
Yes & $14(24.1)$ \\
None & $24(41.4)$ \\
Prior systemic treatment & $8(13.8)$ \\
None & $26(44.8)$ \\
Immunotherapy & \\
Molecular targeted therapy & $19(32.8)$ \\
Number of metastatic sites & $19(32.8)$ \\
1 & $20(34.4)$ \\
2 & $6(10.4)$ \\
3 or more & $42(72.4)$ \\
MSKCC risk group & $10(17.2)$ \\
Favorable & Oncology Group; \\
Intermediate & Poor
\end{tabular}

When the patients tolerated the treatment and their conditions were stable, laboratory tests were performed only on day 1 of each cycle. In the first cycle of treatment, physical examination was also performed on day 14 .

\subsection{Safety and Efficacy Criteria}

The toxicity grade of AEs was defined according to the National Cancer Institute Common Toxicity Criteria, Version 3.0. The usual dose interruption or reduction scheme was used. If Grade 3 hematologic toxicities occurred, treatment was withheld until recovery to Grade 2 or less, at which time sunitinib treatment was resumed at the same dose. In the case of Grade 4 hematologic or Grade 3/4 non-hematologic toxicities, treatment was withheld until those patients achieved at least recovery to Grade 2 or less, at which time the dose was reduced (50 to $37.5 \mathrm{mg}$ daily, 37.5 to $25 \mathrm{mg}$ daily, or 25 to $12.5 \mathrm{mg}$ daily).

\subsection{Follow-up}

CT was performed to examine tumor size and new lesions at baseline and after every 2 cycles or when indicated clinically. Treatment was continued until disease progression or AEs. Response of the treatment was evaluated on the basis of tumor images obtained by CT and other techniques, laboratory data, and subjective/objective symptoms, as well as signs before, during, and after administration of the study drug and during the 
period from completion of treatment to final analysis. Evaluation was performed in compliance with the Response Evaluation Criteria in Solid Tumors (RECIST [v.1.0]) Guidelines for antitumor activities [17].

\subsection{Statistical Analyses}

Time to treatment failure (TTF) was calculated from the start of treatment to the date of the first evidence of disease progression or to the date of discontinuing treatment due to AEs. Progression free survival (PFS) was calculated from the start of treatment to the date of the first evidence of disease progression. OS was calculated from the start of treatment to death or to the last follow-up. The Kaplan-Meier method [18] was used to analyze all time-event variables, with differences between curves analyzed using the log-rank test.

\section{Results}

\subsection{Efficacy of Sunitinib Treatment}

Best tumor responses to sunitinib are listed in Table 2. Partial response (PR), stable disease (SD), and progressive disease (PD) as best objective response were observed in 13 (22.4\%), $26(44.8 \%)$, and 19 (32.8\%) patients, respectively, but complete response was not observed, according to RECIST criteria. The disease control rate $(\mathrm{PR}+\mathrm{SD})$ was $67.2 \%$. In the prior nephrectomy group, PR, SD, and PD were observed in 13 (29.6\%), 21 (47.7\%), and $10(22.7 \%)$ patients, respectively. On the other hand, in the biopsy group without prior nephrectomy, PR, SD, and PD were observed in 0, 5 (35.7\%), and 9 $(64.3 \%)$ patients, respectively. The median observation time from sunitinib initiation was 6.6 months (range, 0.77 - 27.5). Twenty-seven patients died due to cancer progression during this follow-up period. The median TTF was 5.4 (95\% confidence interval [CI]: $2.83,10.2)$ months (Figure 1(a)), PFS was 5.8 (95\% CI: 3.17, 10.2) months (Figure 1(b)), and the median OS was $11.2(95 \%$ CI: 7.2, 21.7) months (Figure 1(c)). In the prior nephrectomy group, the median TTF was 9.0 (95\% CI: $5.43,11.6)$ months (Figure 2(a)), and the median OS was 16.4 (95\% CI: 11, not reached) months (Figure 2(b)). In the non-nephrectomy group, the median TTF was 1.1 (95\% CI: 0.47, 2.53) months (Figure 2(a)), and the median OS was 2.8 (95\% CI: 1.56, 8.37) months (Figure 2(b)). TTF and OS were significantly different between these 2 groups $(\mathrm{P}<0.0001$ and $\mathrm{P}<0.0001$, respectively). Interestingly, MSKCC risk criteria were significant predictors not only of the prognosis, but also the efficacy, of sunitinib. There were significant differences in TTF (favorable risk versus poor risk; $\mathrm{P}=0.0065$, intermediate risk versus poor risk; $\mathrm{P}=0.0123$, Figure3(a)) and OS (favorable risk versus poor risk; $\mathrm{P}=0.0057$, intermediate risk
Table 2. Best tumor response by sunitinib treatment.

\begin{tabular}{cccc}
\hline \multirow{2}{*}{ Response } & $\begin{array}{c}\text { Overall } \\
\mathrm{n}=58\end{array}$ & $\begin{array}{c}\text { Prior nephrecto- } \\
\text { myn }=44\end{array}$ & $\begin{array}{c}\text { Non-nephrectomy } \\
\mathrm{n}=14\end{array}$ \\
\cline { 2 - 4 } & $(\%)$ & $(\%)$ & $(\%)$ \\
\hline CR & 0 & 0 & 0 \\
PR & $13(22.4)$ & $13(29.6)$ & 0 \\
SD & $26(44.8)$ & $21(47.7)$ & $5(35.7)$ \\
PD & $19(32.8)$ & $10(22.7)$ & $9(64.3)$ \\
DCR & $39(67.2)$ & $34(77.3)$ & $5(35.7)$
\end{tabular}

Abbreviation: CR, complete response; PR, partial response; SD, stable disease; PD progressive disease; DCR, disease control rate.

versus poor risk; $\mathrm{P}=0.0007$, Figure3(b)) between MSKCC risk groups.

We also examined the relationship between the dosage of sunitinib administered and the efficacy of treatment. We divided patients into 2 groups according to the mean dose of sunitinib administered over the entire treatment course, i.e., higher or lower than the median dosage (27 $\mathrm{mg}$ daily). The low-dose group displayed a greater median OS than the high-dose group (21.7 months vs. 7.1 months, $\mathrm{P}=0.029)$. During the course of treatment, many patients required a dose reduction $(n=41,70.7 \%)$. As the number of treatment cycles increased, the average dosage of sunitinib decreased significantly $(\mathrm{P}=0.018)$.

No significant relationship was observed between TTF or OS and prior treatment history, number of metastatic sites, and pathologic subtypes.

\subsection{Evaluation of AEs}

AEs that occurred in more than $10 \%$ of patients receiving sunitinib therapy are shown in Table 3. All 58 patients $(100 \%)$ experienced 1 or more AEs. In total, 40 of the 58 patients $(70.0 \%)$ experienced any Grade $3 / 4 \mathrm{AE}$. No fatal Grade 5 AEs were observed. The most frequently occurring Grade 3/4 treatment-related AEs and laboratory abnormalities were anorexia (17.2\%), fatigue (12.1\%), thrombocytopenia (13.8\%), and anemia (12.1\%). Two patients could not continue sunitinib treatment due to severe AEs, namely, diarrhea and thrombocytopenia. Although the frequency was low, we observed clinically important Grade 3 treatment-related AEs, including a decline in left ventricular ejection fraction (1 case), tumor hemorrhage (1 case), and adrenal insufficiency (2 cases). Hypothyroidism was also considered to be an important $\mathrm{AE}$ associated with sunitinib treatment, for which 31 patients $(53.4 \%)$ required medication during this study. 


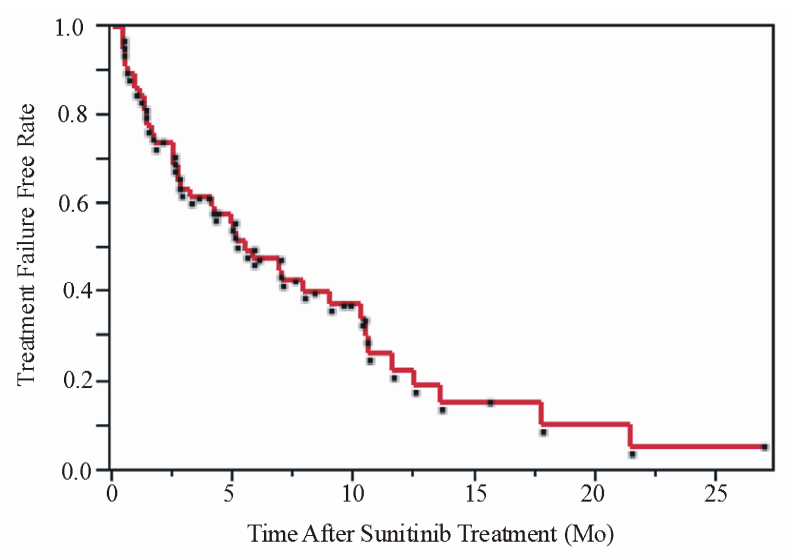

a)

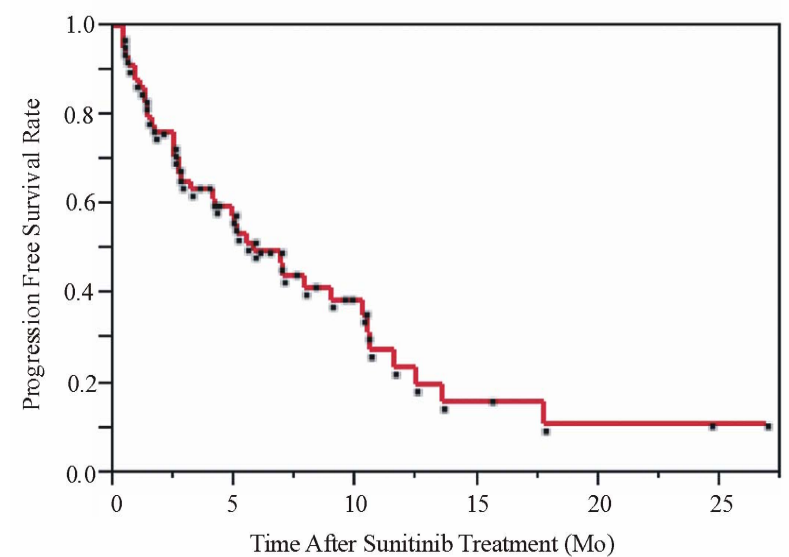

(b)

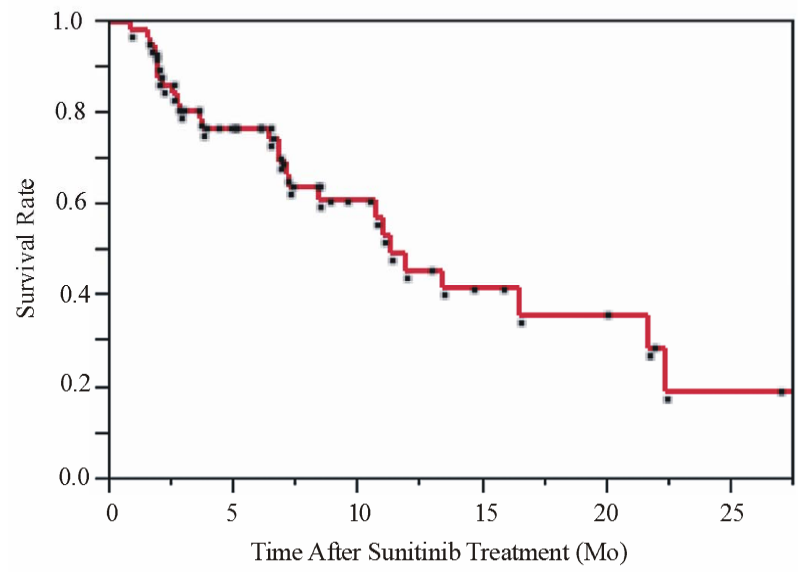

(c)

Figure 1. Kaplan-Meier estimates for time to treatment failure (a), progression-free survival (b), and overall survival (c) in all patients.

\section{Discussion}

IFN- $\alpha$ and/or interleukin- 2 constituted the main therapy for $\mathrm{mRCC}$ before the appearance of molecular-targeted drugs. Recently, the main therapy for $\mathrm{mRCC}$ has shifted to molecular-targeted drugs such as sorafenib and sunitinib. Although many clinical trials have assessed the ef-

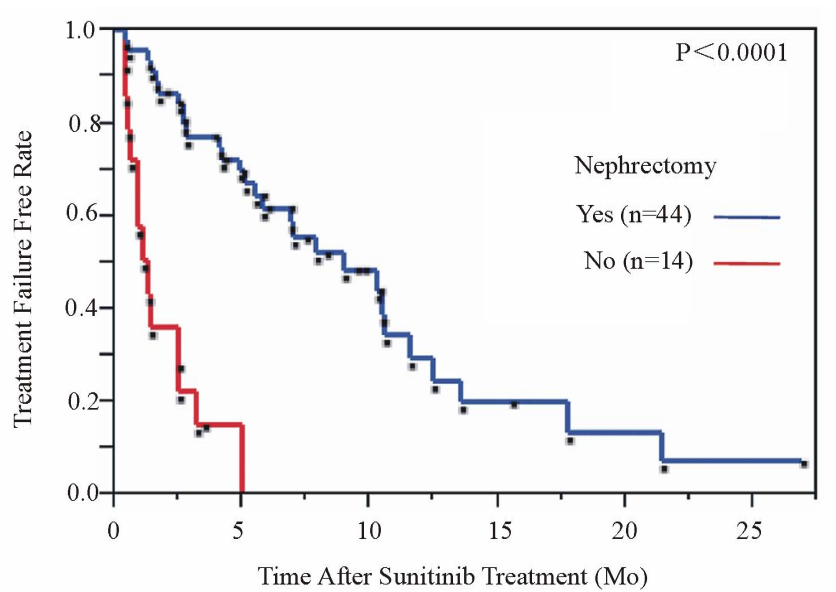

(a)

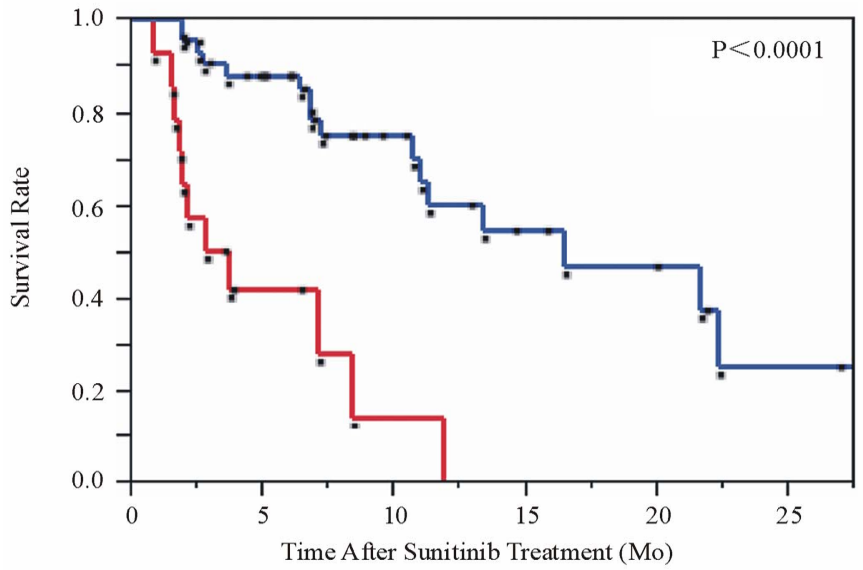

(b)

Figure 2. Kaplan-Meier estimates for time to treatment failure (a) and overall survival (b) in the prior nephrectomy population (blue bar) and the non-nephrectomy population (red bar).

ficacy and safety of these agents in the Western world $[7,8,11]$, only few reports have described findings in Asian populations [12-14,19].

In 2010, Tomita et al. reported updated results from a Phase II trial of sunitinib in the Japanese population [14]. In this report, the median PFS and OS for firstline-treated patients were 12.2 months and 33.1 months, respectively. In contrast, our analysis showed a median PFS of 5.4 months and OS of 11.2 months. The difference between these two studies may have arisen from the differences in patient backgrounds. The participants of the Phase II study were screened and selected, and only treatment-naïve patients and those receiving first-line treatment with molecular-targeted therapy were included; further, all patients had undergone previous nephrectomy and were diagnosed with clear-cell RCC. In contrast, our group of patients appears to reflect more accurately the types of patient encountered in daily clinical practice.

In this study of sunitinib in Japanese patients with 


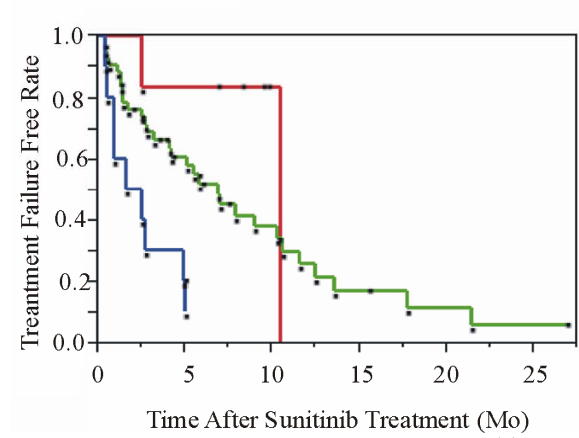

MSKCC risk criteria

favorable risk $(\mathrm{n}=6)$

intermediate risk $(\mathrm{n}=42)$

poor risk $(\mathrm{n}=10)$

favorable risk v.s.poor risk;

$\mathrm{P}=0.0065$

favorable risk v.s.intermediate risk;

$\mathrm{P}=0.2348$

intermediate risk v.s.poor risk;

$\mathrm{P}=0.0123$

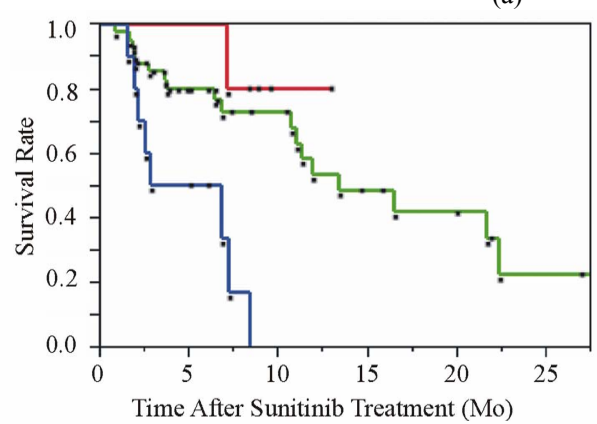

favorable risk v.s.poor risk;

$\mathrm{P}=0.0057$

favorable risk v.s.intermediate risk;

$\mathrm{P}=0.4234$

intermediate risk v.s.poor risk;

$\mathrm{P}=0.0007$

(b)

Figure 3. Kaplan-Meier estimates for time to treatment failure (a) and overall survival (b) in the favorable risk population (red bar, $n=6$ ), intermediate risk population (green bar, $n=42$ ), and poor risk population (blue bar, $n=10$ ), based on Memorial Sloan-Kettering Cancer Center (MSKCC) criteria.

Table 3. Treatment-related adverse events and laboratory abnormalities reported in more than $10 \%$ of the patients.

\begin{tabular}{|c|c|c|c|c|c|c|}
\hline \multirow{2}{*}{ Adverse event } & \multicolumn{2}{|c|}{ Any Grade } & \multicolumn{2}{|c|}{ Grade 3} & \multicolumn{2}{|c|}{ Grade 4} \\
\hline & No. of patients & $\%$ & No. of patients & $\%$ & No. of patients & $\%$ \\
\hline \multicolumn{7}{|l|}{ Treatment-Related Adverse Event } \\
\hline Anorexia & 40 & 69 & 10 & 17.2 & 0 & 0 \\
\hline Fatigue & 36 & 62.1 & 7 & 12.1 & 0 & 0 \\
\hline Hand-foot syndrome & 34 & 58.6 & 5 & 8.6 & 0 & 0 \\
\hline Skin discoloration & 24 & 41.4 & 0 & 0 & 0 & 0 \\
\hline Diarrhea & 23 & 39.7 & 0 & 0 & 1 & 1.7 \\
\hline Hypertension & 20 & 34.5 & 2 & 3.4 & 0 & 0 \\
\hline Fever & 19 & 32.8 & 0 & 0 & 0 & 0 \\
\hline Stomatitis & 18 & 31 & 0 & 0 & 1 & 1.7 \\
\hline Dysgeusia & 13 & 22.4 & 0 & 0 & 0 & 0 \\
\hline Skin rash & 12 & 20.7 & 0 & 0 & 0 & 0 \\
\hline Constipation & 7 & 12.1 & 0 & 0 & 0 & 0 \\
\hline Vomiting & 7 & 12.1 & 1 & 1.7 & 0 & 0 \\
\hline Edema & 6 & 10.3 & 0 & 0 & 0 & 0 \\
\hline Voice changes & 6 & 10.3 & 0 & 0 & 0 & 0 \\
\hline \multicolumn{7}{|l|}{ Laboratory Abnormality } \\
\hline Thrombocytopenia & 41 & 70.7 & 5 & 8.6 & 3 & 5.2 \\
\hline Leukopenia & 41 & 70.7 & 5 & 8.6 & 0 & 0 \\
\hline Anemia & 34 & 58.6 & 6 & 10.3 & 1 & 1.7 \\
\hline Hypothyroidism & 31 & 53.4 & 0 & 0 & 0 & 0 \\
\hline Increased aspartate aminotransferase & 31 & 53.4 & 4 & 6.9 & 0 & 0 \\
\hline Increased creatinine & 27 & 46.6 & 0 & 0 & 0 & 0 \\
\hline Increased alkaline phosphatase & 21 & 36.2 & 2 & 3.4 & 0 & 0 \\
\hline Hypophosphatemia & 17 & 29.3 & 3 & 5.2 & 0 & 0 \\
\hline Hypocalcemia & 15 & 25.9 & 0 & 0 & 0 & 0 \\
\hline Increased $\gamma$-glutamyl transpeptidase & 15 & 25.9 & 1 & 1.7 & 0 & 0 \\
\hline Increased alanine aminotransferase & 15 & 25.9 & 3 & 5.2 & 0 & 0 \\
\hline Increased lipase & 12 & 20.7 & 1 & 1.7 & 1 & 1.7 \\
\hline Increased amylase & 10 & 17.2 & 2 & 3.4 & 0 & 0 \\
\hline
\end{tabular}


$\mathrm{mRCC}$, the disease control rate was $67.2 \%$ in all patients, $77.3 \%$ in the prior nephrectomy population, and $35.7 \%$ in the non-nephrectomy population. Thus, sunitinib showed significant antitumor activity, particularly in patients with a history of nephrectomy, with a median OS benefit of more than 1 year compared with patients with no history of nephrectomy; treatment was also generally well tolerated. In our study, the non-nephrectomy population included those patients with no indication of surgery at the start of treatment, namely, very rapid growth with multiple metastases at the first examination and poor performance status among others. Our results indicate that the effect of sunitinib is very limited in such a population. For such patients, temsirolimus may be a good alternative treatment option [20]. In this study, we also found a significant difference between MSKCC risk groups for TTF and OS. Thus, MSKCC risk score was found to be a good marker of survival and the efficacy of treatment not only for the Western population, but also for the Japanese population with $\mathrm{mRCC}$.

In this study, we did not observe a linear relationship between sunitinib dosage and the efficacy of treatment. Patients receiving longer treatment tended to require a dose reduction due to AEs, and we found a significant negative relationship between the number of treatment cycles and sunitinib dosage. This finding explains why the lower dose of sunitinib was associated with longer survival. Our results indicate that patient-related factors may be more important than sunitinib dosage to predict the efficacy of treatment. However, larger and prospective studies are necessary to more accurately assess the relationship between dosage and sunitinib efficacy.

Safety findings from the present study indicate that the safety profile of sunitinib is generally similar in Asian and non-Asian patients. Although Grade 3/4 AEs were observed in nearly $70 \%$ of cases in our study, most of these AEs were manageable with pre-specified dose changes or standard medical treatment.

Compared with sorafenib [19], the incidence of hypothyroidism associated with sunitinib treatment was significantly higher ( $21 \%$ and $53 \%$, respectively). Thus, the physician should carefully assess thyroid function before and during sunitinib treatment. In this study, the most frequently reported Grade 3/4 treatment-related AEs and laboratory abnormalities were anorexia, fatigue, hand-foot syndrome, thrombocytopenia, anemia, and leukopenia. Grade 3/4 thrombocytopenia, anemia, and leukopenia were considerably more common in Japanese patients than in Western patients. Further studies are necessary to validate and investigate the basis of potential sunitinib tolerability and efficacy differences between these patient populations. In this respect, it may be interesting to assess the relationship between genetic background and efficacy and toxicity profiles of sunitinib in different patient groups.

\section{Conclusions}

Sunitinib had a favorable risk/benefit profile in Japanese mRCC patients with a history of nephrectomy. The safety of sunitinib was similar to that reported in Western studies, although there was an increased incidence of hematologic AEs in Japanese patients.

\section{Acknowledgements}

There are no conflicts of interest, including specific financial interests and relationships and affiliations relevant to the subject matter or materials discussed in the manuscript (e.g., employment/affiliation, grants or funding, consultancies, honoraria, stock ownership or options, expert testimony, royalties or patents field, received or pending) to be disclosed.

\section{REFERENCES}

[1] D. M. Parkin, F. Bray, J. Ferlay and P. Pisani, "Global Cancer Statistics, 2002," A Cancer Journal for Clinicians, Vol. 55, No 2, 2005, pp. 74-108. doi:10.3322/canjclin.55.2.74

[2] R. J. Motzer, N. H. Bander and D. M. Nanus, "RenalCell Carcinoma," The New England Journal of Medicine, Vol. 335, No. 12, 1996, pp. 865-875. doi:10.1056/NEJM199609193351207

[3] C. Coppin, F. Porzsolt, A. Awa, J. Kumpf, A. Coldman and T. Wilt, "Immunotherapy for Advanced Renal Cell Cancer," Cochrane Database of Systematic Reviews, 2005, Vol. 1, p. 1425.

[4] X. I. Na, G. Wu, C. K. Ryan, S. R. Schoen, P. A. di'Santagnese and E. M. Messing, "Overproduction of Vascular Endothelial Growth Factor Related to von Hippel-Lindau Tumor Suppressor Gene Mutations and Hypoxia-Inducible Factor-1alpha Expression in Renal Cell Carcinoma," Journal of Urology, Vol. 170, No. 2 Part 1, 2003, pp. 588-592.

[5] B. I. Rini and E. J. Small, "Biology and Clinical Development of Vascular Endothelial Growth Factor-Targeted Therapy in Renal Cell Carcinoma," Journal of Clinical Oncology, Vol. 23, No. 5, 2005, pp. 1028-1043. doi:10.1200/JCO.2005.01.186

[6] B. Escudier, A. Pluzanska, P. Koralewski, A. Ravaud, S. Bracarda, C. Szczylik, C. Chevreau, M. Filipek, B. Melichar, E. Bajetta, V. Gorbunova, J. O. Bay, I. Bodrogi, A. Jagiello-Gruszfeld, N. Moore and AVOREN Trial Investigators, "Bevacizumab Plus Interferon Alfa-2a for Treatment of Metastatic Renal Cell Carcinoma; a Randomized, Double-Blind Phase III Trial," Lancet, Vol. 370, No. 9605, 2007, pp. 2103-2111. doi:10.1016/S0140-6736(07)61904-7

[7] B. Escudier, T. Eisen, W. M. Stadler, C. Szczylik, S. Oudard, M. Siebels, S. Negrier, C. Chevreau, E. Solska, 
A. A. Desai, F. Rolland, T. Demkow, T. E. Hutson, M. Gore, S. Freeman, B. Schwartz, M. Shan, R. Simantov, R. M. Bukowski, et al., "Sorafenib in Advanced Clear-Cell Renal-Cell Carcinoma," The New England Journal of Medicine, Vol. 356, No. 2, 2007, pp. 125-134. doi.:10.1056/NEJMoa060655

[8] R. J. Motzer, T. E. Hutson, P. Tomczak, M. D. Michaelson, R. M. Bukowski, O. Rixe, S. Oudard, S. Negrier, C. Szczylik, S. T. Kim, I. Chen, P. W. Bycott, C. M. Baum and R. A. Figlin, "Sunitinib versus Interferon Alfa in Metastatic Renal-Cell Carcinoma," The New England Journal of Medicine, Vol. 356, No. 2, 2007, pp. 115-124. doi:10.1056/NEJMoa065044

[9] D. B. Mendel, A. D. Laird, X. Xin, S. G. Louie, J. G. Christensen, G. Li, R. E. Schreck, T. J. Abrams, T. J. Ngai, L. B. Lee, L. J. Murray, J. Carver, E. Chan, K. G. Moss, J. O. Haznedar, J. Sukbuntherng, R. A. Blake, L. Sun, C. Tang, T. Miller, S. Shirazian, G. McMahon and J. M. Cherrington, "In Vivo Antitumor Activity of SU11248, a Novel Tyrosine Kinase Inhibitor Targeting Vascular Endothelial Growth Factor and Platelet-Derived Growth Factor Receptors: Determination of a Pharmacokinetic/Pharmacodynamic Relationship," Clinical Cancer Research, Vol. 9, No. 1, 2003, pp. 327-337.

[10] T. J. Abrams, L. B. Lee, L. J. Murray, N. K. Pryer and J. M. Cherrington, "SU11248 Inhibits KIT and Platelet- Derived Growth Factor Receptor Beta in Preclinical Models of Human Small Cell Lung Cancer," Molecular Cancer Therapeutics, Vol. 2, No. 5, 2003, pp. 471-478.

[11] R. J. Motzer, T. E. Hutson, P. Tomczak, M. D. Michaelson, R. M. Bukowski, S. Oudard, S. Negrier, C. Szczylik, R. Pili, G. A. Bjarnason, X. Garcia-del-Muro, J. A. Sosman, E. Solska, G. Wilding, J. A. Thompson, S. T. Kim, I. Chen, X. Huang and R. A. Figlin, "Overall Survival and Updated Results for Sunitinib Compared with Interferon Alfa in Patients with Renal Cell Carcinoma," Journal of Clinical Oncology, Vol. 27, No. 22, 2009, pp. 3584-3590. doi:10.1200/JCO.2008.20.1293

[12] C. Yoo, J. E. Kim, J. L. Lee, J. H. Ahn, D. H. Lee, J. S. Lee, S. Na, C. S. Kim, J. H. Hong, B. Hong, C. Song and H. Ahn, "The Efficacy and Safety of Sunitinib in Korean Patients with Advanced Renal Cell Carcinoma: High Incidence of Toxicity Leads to Frequent Dose Reduction," Japanese Journal of Clinical Oncology, Vol. 40, No. 10, 2010, pp. 980-985. doi:10.1093/jjco/hyq073

[13] H. Uemura, N. Shinohara, T. Yuasa, Y. Tomita, H. Fujimoto, M. Niwakawa, S. Mugiya, T. Miki, N. Nonomura, M. Takahashi, Y. Hasegawa, N. Agata, B. Houk, S. Naito and H. Akaza, "A Phase II Study of Sunitinib in Japanese Patients with Metastatic Renal Cell Carcinoma: Insight into the Treatment, Efficacy and Safety," Japanese Jour- nal of Clinical Oncology, Vol. 40, No. 3, 2010, pp. 194-202. doi:10.1093/ijco/hyp146

[14] Y. Tomita, N. Shinohara, T. Yuasa, H. Fujimoto, M. Niwakawa, S. Mugiya, T. Miki, H. Uemura, N. Nonomura, M. Takahashi, Y. Hasegawa, N. Agata, B. Houk, S. Naito and H. Akaza, "Overall Survival and Updated Results from a Phase II Study of Sunitinib in Japanese Patients with Metastatic Renal Cell Carcinoma," Japanese Journal of Clinical Oncology, Vol. 40, No. 12, 2010, pp. 1166-1172. doi:10.1093/jico/hyq146

[15] Y. Horikawa, N. Tsuchiya, K. Yuasa, S. Narita, M. Saito, K. Takayama, T. Nara, H. Tsuruta, T. Obara, K. Numakura, S. Satoh and T. Habuchi, "Efficacy and Safety of Sunitinib for Metastatic Renal Cell Carcinoma: Early Clinical Experiences at Akita University Hospital (Japan)," Japanese Journal of Clinical Oncology, Vol. 41, No. 3, 2011, pp. i6-i17. doi:10.1093/jjco/hyq254

[16] R. J. Motzer, J. Bacik, L. H. Schwartz, V. Reuter, P. Russo, S. Marion and M. Mazumdar, "Prognostic Factors for Survival in Previously Treated Patients with Metastatic Renal Cell Carcinoma," Journal of Clinical Oncology, Vol. 22, No. 3, 2004, pp. 454-463. doi:10.1200/JCO.2004.06.132

[17] P. Therasse, S. G. Arbuck, E. A. Eisenhauer, J. Wanders, R. S. Kaplan, L. Rubinstein, J. Verweij, M. Van Glabbeke, A. T. van Oosterom, M. C. Christian and S. G. Gwyther, "New Guidelines to Evaluate the Response to Treatment in Solid Tumors. European Organization for Research and Treatment of Cancer, National Cancer Institute of the United States, National Cancer Institute of Canada," Journal of the National Cancer Institute, Vol. 92, No. 3, 2000, pp. 205-216. doi:10.1093/jnci/92.3.205

[18] E. L. Kaplan and P. Meier, "Nonparametric Estimation from Incomplete Observations," Journal of the American Statistical Association, Vol. 53, No. 282, 1958, pp. 457481. doi: $10.2307 / 2281868$

[19] T. Ueda, Y. Imamura, A. Komaru, S. Fukasawa, T. Sazuka, T. Suyama, Y. Naya, N. Nihei, T. Ichikawa and M. Maruoka, "Treatment Outcomes of Sorafenib for First Line or Cytokinerefractory Advanced Renal Cell Carcinoma in Japanese Patients," International Journal of Urology, Vol. 17, No. 9, 2010, pp. 811-815.

[20] G. Hudes, M. Carducci, P. Tomczak, J. Dutcher, R. Figlin, A. Kapoor, E. Staroslawska, J. Sosman, D. McDermott, I Bodrogi, Z. Kovacevic, V. Lesovoy, I. G. Schmidt-Wolf, O. Barbarash, E. Gokmen, T. O'Toole, S. Lustgarten, L. Moore, R. J. Motzer and Global ARCC Trial, "Temsirolimus, Interferon Alfa, or Both for Advanced Renal-Cell Carcinoma," The New England Journal of Medicine, Vol. 356, No. 22, 2007, pp. 2271-2281. doi:10.1056/NEJMoa066838 